\title{
Les Moires entre la naissance et la mort : de la représentation au culte
}

\section{Vinciane Pirenne-Delforge et Gabriella Pironti}

\section{(2) OpenEdition}

1 Journals

Édition électronique

URL : http://journals.openedition.org/edl/143

DOI : $10.4000 /$ edl. 143

ISSN : 2296-5084

Éditeur

Université de Lausanne

\section{Édition imprimée}

Date de publication : 15 décembre 2011

Pagination : 93-114

ISBN : 978-2-940331-26-0

ISSN : 0014-2026

\section{Référence électronique}

Vinciane Pirenne-Delforge et Gabriella Pironti, « Les Moires entre la naissance et la mort : de la représentation au culte », Études de lettres [En ligne], 3-4 | 2011, mis en ligne le 15 décembre 2014, consulté le 18 décembre 2020. URL : http://journals.openedition.org/edl/143 ; DOI : https://doi.org/ 10.4000/edl. 143

\section{(C) Études de lettres}




\section{LES MOIRES ENTRE LA NAISSANCE ET LA MORT: DE LA REPRÉSENTATION AU CULTE}

En Grèce ancienne, les Moires sont les puissances divines de la "part» qui revient à chacun et les récits mythiques qui les mettent en scène les associent de façon privilégiée à la naissance et à la mort. Dans leurs figures traditionnelles de fileuses et de tisserandes, ces déesses président au déroulement de la vie de chacun et aux dessins variés qui constituent la trame de la vie. Quant aux attestations de culte rendu aux Moires, elles sont peu nombreuses et peu explicites sur les attentes des fidèles qui les honoraient. Toutefois, les mythes et les rites ne sont pas des ensembles étanches, mais des langages spécifiques qui entrent en résonance dans l'univers mental de ceux qui racontaient les récits et accomplissaient les gestes rituels. Ces deux aspects du savoir grec sur les Moires sont donc étroitement liés et étudiés comme tels dans la présente analyse. Puissances de distribution, de rétribution et de régulation, les Moires sont solidaires de l'équilibre garanti par l'autorité souveraine de Zeus sur un plan mythique et contribuent tout autant à un tel équilibre au sein des communautés humaines.

\section{Introduction}

Dans l'imaginaire des anciens Grecs, il existe des créatures plus ou moins hostiles, plus ou moins monstrueuses selon les cas, qui se penchent sur la vie du jeune enfant, la protègent ou la menacent. Ces figures, qui affleurent rarement dans la documentation, trouvent leur raison d'être dans les peurs liées aux dangers de la naissance et à la fragilité de la petite enfance. De tels croque-mitaines ont été étudiés ${ }^{1}$; ils peuplent un certain imaginaire fantasmatique, mais n'appartiennent pas aux

I. M. Patera, "Comment effrayer les enfants». 
conceptions religieuses des Grecs et à la représentation du monde que ces conceptions contribuent à dessiner. La différence entre la catégorie des croque-mitaines et celle que cet article aborde est simple à établir, à défaut d'être simple à étudier: les figures de la première catégorie, que l'on peut qualifier de "folkloriques», n'apparaissent pas dans les mythes cosmogoniques et théogoniques, et, surtout, elles ne font l'objet d'aucun culte établi. C'est donc vers le panthéon grec que l'analyse s'orientera dans la réflexion qui suit.

Ce sont les Moires qui correspondent le mieux aux deux critères évoqués: la présence autour des jeunes enfants et le profil divin. Toutefois, il convient de nuancer d'emblée une certaine vision des Moires, celle des dictionnaires de mythologie, qui informe prioritairement la vulgate les concernant: c'est l'image de trois inflexibles déesses, maîtresses du destin, qui orientent l'existence humaine à la naissance et en coupent matériellement le fil une fois son terme achevé. Comme toutes les vulgates, celle-ci n'est ni totalement fausse ni entièrement satisfaisante, et donne le sentiment confortable d'avoir compris ces figures divines une fois qu'un tel portrait «canonique» en a été dressé.

Or le système polythéiste des anciens Grecs est un ensemble plus complexe que ne laisse entendre une certaine vision stéréotypée, pour une large part héritée de la Renaissance. L’ensemble complexe en question est comme un organisme vivant, dont toutes les parties sont reliées entre elles et contribuent au bon fonctionnement global. Chaque partie - en l'occurrence chaque dieu - a un rôle à jouer, un rôle spécifique, mais dont la définition doit éviter le piège de la simplification. Un dieu grec est une puissance dont les applications échappent souvent à notre logique moderne car elles font résonner des données que nous avons tendance à séparer ${ }^{2}$. Le déterminisme culturel est par excellence le piège à éviter.

Pour l'éviter, précisément, il faut tenter de saisir une figure divine dans sa cohérence au fil des récits et des rituels. C'est de cette manière que nous souhaitons évaluer la manière dont les Moires interviennent dans le champ de l'accouchement et des naissances. L'articulation du propos sera donc assez simple: après une lecture de quelques traditions littéraires qui mettent les Moires en scène, nous nous pencherons sur les

2. Voir les analyses de G. Pironti, Entre ciel et guerre, à propos de la déesse Aphrodite. 
cultes qu'elles recevaient, afin de repérer les éléments constitutifs de leur puissance divine.

Une telle entreprise fait partie d'un projet plus large, qui consiste à mieux circonscrire le fonctionnement du polythéisme en évitant deux écueils : celui qui consiste à faire du panthéon un indescriptible fouillis au sein duquel les Grecs opéraient des choix au total assez aléatoires et celui qui associe chaque dieu à une fonction précise et définie de façon si étroite et si statique qu'elle entre en contradiction avec ce que rapportent certaines sources. Ces deux positions ne rendent justice ni à l'intelligence des Grecs ni à la longévité du système religieux en question. C'est entre ces deux extrêmes que se situe l'analyse qui suit, faisant le pari de la cohérence des figures divines, sans en figer la détermination.

\section{Au fil de la vie: les Moires et la naissance dans les récits mythiques}

En Grèce ancienne, le moment crucial qu'est la naissance d'un enfant est placé sous la protection de nombreuses divinités qui, selon les circonstances et les contextes, prennent en charge l'un ou l'autre aspect de ce processus complexe. Comme le choix de telle ou telle divinité n'est pas purement gratuit, il faut comprendre de quelle manière chaque dieu intervient au cœur du processus. Or le nom même des Moires, qui renvoie à moira, "la part", donne une première indication. Dans le processus de la naissance, les Moires président à la "part» qui revient à chacun. Dans d'autres domaines aussi, elles s'occupent de répartition et de partage. La répartition, qui est essentiellement un partage de pouvoirs et de compétences, est un acte fondamental en Grèce ancienne, qui fonde la société des dieux ainsi que celle des hommes ${ }^{3}$. Les Moires sont à la fois des figures liées à la répartition et à l'ordonnancement, de même que des puissances d'inauguration et d'accomplissement.

Dans le domaine précis qu'est la naissance d'une nouvelle vie, les récits mythiques déclinent leurs compétences de manière différente selon la nature mortelle ou immortelle de l'enfant dont elles sanctionnent l'entrée dans le monde. S’agissant de la naissance d'un dieu, cette "part"

3. G. Pironti, «Dans l'entourage de Thémis». Voir également M.-Ch. Leclerc, «Le partage des lots». Sur ce partage mis en œuvre par les divinités qui président à la naissance, voir aussi dans ce volume l'article de C. Frank, «Le fuseau et la quenouille». 
tend à valoriser à la fois la nature immortelle de l'enfant et la position que la nouvelle puissance divine va occuper dans la société des dieux ${ }^{4}$. Mais quand il s'agit d'un être humain, la "part" en question est marquée par la condition mortelle du nouveau-né et se présente tout d'abord comme le segment de vie qui lui est attribué, avec un début et une fin. Les Moires veillent au respect de ces limites, qui est inhérent au cycle vital lui-même.

Les historiens des religions ont longtemps privilégié l'image des Moires en tant que déesses de la mort dont la fonction originelle serait d'imposer à chaque homme le cruel destin qui lui est réservé 5 . En effet, dans l'épopée homérique, le nom commun moira est souvent synonyme de "mort». La prégnance d'une telle image est aisément explicable: tout d'abord, la mort délimite et définit la "part» des hommes, mais aussi, dans un cadre littéraire qui exalte la mort héroïque, c'est le côté sombre de cette part qui prévaut. L'image de la moira cruelle qui s'empare d'un individu devient ainsi l'une des représentations de la mort les plus utilisées dans la poésie grecque et dans les inscriptions funéraires ${ }^{6}$.

Toutefois, le succès de cette image ne doit pas conduire à négliger les autres emplois du nom commun moira qui, dans le langage épique déjà, exprime aussi l'idée de "part, ordre, mesure, convenance " ${ }^{7}$. Il faut surtout rappeler que les Moires, les «parts» divines, sont associées dans la tradition grecque non seulement à la fin de la vie, mais aussi à ses débuts. En outre, elles président à ce mélange de bien et de mal qui est l'apanage de la condition humaine. Dès lors, elles règlent les vicissitudes

4. Voir, par exemple, Callimaque, Artémis, 20-25, où la déesse supplie son père en ces termes: "J'habiterai les monts, et ne fréquenterai les cités des hommes qu'appelée à l'aide par les femmes que tourmentent les âpres douleurs; les Moires, à l'heure même où je naquis, m'ont assigné de les secourir, car ma mère me porta et m'enfanta sans souffrance, et sans douleur déposa le fruit de ses entrailles.»

5. P. ex. B. C. Dietrich, Death, Fate and the Gods.

6. Sur le terme moira, le rapport entre "part» et "destin», et les Moires, vaste sujet qui dépasse le cadre plus restreint de cette étude, voir aussi W. C. Greene, Moira; U. Bianchi, $\triangle I O \Sigma A I \Sigma A$; C. B. Pistorio, Fato e divinità nel mondo greco; R. B. Onians, Le origini del pensiero europeo, p. 469-492; B. C. Dietrich, Death, Fate and the Gods; A. Magris, L'idea di destino nel mondo antico. Pour une étude récente sur «l'imaginaire du destin» dans la poésie archaïque, voir K. Mackowiak, "De moira aux Moirai, de l'épopée à la généalogie». Voir aussi l'inscription d'Izmir citée à la fin de l'article de V. Dasen, «Le pouvoir des femmes» dans ce volume.

7. Voir G. Di Mauro Battilana, «MOIPA» e «AILA» in Omero, spéc. p. 42-47. 
alternées de la vie des hommes et en scandent les étapes essentielles tel la naissance, le mariage, l'accouchement et la mort. Aucun indice ne permet d'étayer la thèse évolutionniste qui fait de ces compétences le fruit d'un développement secondaire de la figure des Moires à partir de leur prétendue fonction originelle de déesses de la mort. Au contraire, les plus anciens témoignages dont nous disposons à leur sujet, à savoir les poèmes archaïques d'Homère et d'Hésiode, plaident en faveur d'un profil complexe inhérent à ces figures divines dès leur apparition.

La seule attestation du pluriel Moirai dans les poèmes homériques ne met pas les déesses ainsi dénommées en relation avec la mort: c'est Apollon qui prend la parole pour persuader les dieux qu'il est temps qu'Achille rende le cadavre d'Hector; le dieu rappelle à cette occasion que les pleurs et le chagrin pour la mort d'un proche ne peuvent pas rester sans limites et que «les Moires ont doté les hommes de la capacité d'endurer les malheurs" ${ }^{8}$. Selon Hésiode, les Moires sont chargées d'attribuer aux hommes les bienfaits et les malheurs ${ }^{9}$, et Solon développera cette image un bon siècle plus tard, en rappelant la variabilité et l'imprévisibilité de la condition humaine: si parfois les douleurs les plus atroces qu'aucun remède n'a su apaiser jusque-là disparaissent soudainement, dit-il, et que l'on se retrouve, sans savoir comment, dans un état de parfaite santé, c'est que «les Moires apportent aux hommes le mal, mais aussi le bien, et qu'on ne peut se soustraire aux dons des dieux " ${ }^{10}$. Les déesses permettent aux mortels de supporter les malheurs qui les accablent en les compensant de manière positive. Comme le suggère aussi l'Apollon de l'Iliade, les Moires font en sorte que les mortels puissent assumer les vicissitudes de leur imprévisible existence.

Dans la Théogonie d'Hésiode, les Moires occupent deux positions généalogiques différentes qui illustrent chacune des aspects de leurs pouvoirs. Anciennes déesses précédant le royaume de Zeus, les Moires sont filles de la Nuit; dans le funeste lignage nocturne, la Mort prend trois visages différents: tout d'abord celui de Thanatos, l'épuisement définitif des forces vitales, frère du Sommeil, Hypnos, qui représente lui l'épuisement temporaire de ces mêmes forces; mais il y a aussi Kèr au singulier,

8. Homère, Iliade, 24.44-49.

9. Hésiode, Théogonie, 901-906.

Io. Solon, IEG II, fr. 13.59-64 (éd. M. West). 
la "Mort qui tue", et Moros, la "Mort qui échoit à chacun" ${ }^{11}$. Le "lot fatal» prend donc le visage masculin de Moros, qui est ainsi distinct de ses sœurs, les Moires. Ces dernières apparaissent plus loin dans la même généalogie aux côtés de puissances divines justicières et vengeresses telles que Némésis et les Kères ${ }^{12}$.

Mais les Moires réapparaissent aussi plus avant dans ce même poème, en tant que filles de Zeus et de la déesse Thémis, puissance divine de la norme ${ }^{13}$. Dans cette seconde famille, elles reçoivent alors comme sœurs les Hôrai, les Heures, déesses qui président aux circonstances propices pour l'épanouissement de la vie sous toutes ses formes. De ces Moires, qui donnent aux hommes tant le bien que le mal, Hésiode précise les noms: elles s'appellent Klotho, la «Fileuse», Lachésis, "Celle qui préside à l'attribution du sort» et Atropos, «l'Inflexible».

Il n'y a pas de Moires maléfiques, filles de Nuit, auxquelles s'opposeraient des Moires bénéfiques, filles de Zeus. En fait, les deux généalogies coopèrent pour dessiner le profil complexe de ces divinités. Leur puissance normative est exprimée par la relation avec la sphère de la themis, leur lien avec la promotion et la régulation du cycle vital par la parenté avec les Heures. Leur place dans la généalogie nocturne signale en revanche l'aspect nécessaire et redoutable du pouvoir des Moires qui non seulement sont chargées de promouvoir l'ordre des choses, mais aussi de le garantir et de le rétablir s'il a été violé.

Les Moires déroulent le fil de la vie de chacun du début à la fin. Dès les poèmes homériques, ces déesses se retrouvent associées à la naissance aussi bien qu'à la mort d'un individu: les hommes, au moment de leur mort, - dit le poète - ne font qu'obéir à ce que la Moire, ou les "Fileuses", les Klôthes, ont filé pour eux lors de l'accouchement ${ }^{14}$. Le contexte épique, parcouru par le thème de la mort hérö̈que, colore sans doute de manière un peu sombre la présence des Moires aux côtés des nouveau-nés. Mais derrière cette nuance, on entrevoit l'image "neutre", pour ainsi dire, des déesses qui déroulent le fil de la vie, en inaugurant cette opération à la naissance de l'individu. "Fileuses» au moment de

II. Hésiode, Théogonie, 211-212.

I2. Hésiode, Théogonie, 217-224.

I3. Hésiode, Théogonie, 901-906. Voir M. Corsano, Themis; J. Rudhardt, Thémis et les Hôrai; G. Pironti, «Dans l'entourage de Thémis».

I4. Homère, Iliade, 24.209-210; Homère, Odyssée, 7.197. Voir également Homère, Iliade, 20.127-128. 
l'accouchement plutôt que "tisserandes", comme elles peuvent l'être à d'autres occasions ${ }^{15}$, les Moires traduisent par ce geste une certaine représentation de la naissance: tout se passe comme si elles étaient chargées de démêler un amas indistinct pour en extraire un fil précis, censé correspondre à l'identité du nouveau-né et qui évoque sans doute "sa part", sa moira, à savoir à la fois le segment de vie qui lui sera attribué et la place qu'il va occuper, pendant ce temps, dans la communauté des siens.

La filature des événements qui ponctuent la vie des hommes est attribuée aussi à l'ensemble des dieux, mais le début du processus, avec l'opération de démêlage nécessaire au déroulement du fil de la vie lui-même, semble bien être l'apanage des Moires. On comprend mieux dès lors l'association étroite, dans la tradition grecque, de ces déesses avec Ilithyie, puissance divine dont la présence est indispensable lors de l'accouchement d'une nouvelle vie ${ }^{16}$. A cette occasion, un processus s'accomplit, la gestation d'une nouvelle existence, et un processus commence, la vie de l'enfant: les Moires sont bien à leur place aux côtés d'Ilithyie pour sanctionner ce double passage. Chez Pindare, on retrouve Moires et Ilithyie présidant ensemble à la naissance d'Iamos, le fils d'Apollon ${ }^{17}$. Selon un fragment attribué à ce même poète, lors de la naissance d'Apollon et de sa sœur Artémis, Ilithyie et Lachésis, l'une des Moires, lancèrent ensemble le cri rituel célébrant la naissance des jumeaux divins ${ }^{18}$. Mais leur association n'est pas une invention du poète thébain: dans un ancien hymne délien à Ilithyie, mentionné par Pausanias, la déesse est qualifiée d'eulinos et associée par cette épithète à l'action de dérouler le fil, qui est d'habitude le propre des Moires ${ }^{19}$. Le recoupement de compétences fonctionne aussi dans l'autre sens: suivant le récit de la naissance

15. Voir, par exemple, Fragmenta Adespota, fr. 1018, a-b (éd. D. Page, Poetae Melici Graeci, Oxford, 1963).

I6. Pindare, Néménnes, 7.1-4: «Ilithyie, assistante des Moires aux pensées profondes, fille d'Héra la puissante, écoute, génitrice des enfants! Sans toi nous ne verrions pas le jour, ni l'heure bienfaisante des ténèbres; sans toi nous ne posséderions pas ta sœur, Hébé ["Jeunesse»] aux membres splendides. "Voir S. Pingiatoglou, Eileithyia et M. Bettini, Nascere, spéc. p. 92-101.

I7. Pindare, Olympiques, 6.41-44: «Le dieu aux cheveux d'or mit près d'elle [Evadne, fille de Poséidon et Pitané] Ilithyie et les Moires bienveillantes; et de ses flancs, par un doux travail, Iamos vint au monde, aussitôt.»

I8. Pindare, Paeanes, 12.14-18 (éd. H. Maehler, Teubner, 1989).

I9. Pausanias, 8.21.3-4. 
d'Asclépios que transmet une inscription métrique d'Epidaure, Apollon confie la venue au monde de son fils aux soins des Moires et surtout de Lachésis, qualifiée pour l'occasion de "noble sage-femme» ${ }^{20}$. Si l'on considère l'ensemble des attestations des Moires dans les poèmes de Pindare, on les voit tantôt associées au mariage ${ }^{21}$, tantôt à l'accomplissement des vœux formulés ${ }^{22}$, tantôt à la naissance, et cela de plusieurs manières. Klotho est représentée, par exemple, dans le geste de sortir du bassin lustral le corps reconstitué de Pélops, l'enfant qui avait été tué et mis en pièces par son père, mais que les dieux ramènent à une nouvelle $v_{i e}{ }^{23}$. Lachésis de son côté assiste à la naissance de l'île de Rhodes et en sanctionne l'attribution à Hélios, le seul parmi les dieux qui jusquelà n'avait pas encore reçu de terre en partage (lachos) ${ }^{24}$. En outre, chez Pindare, les Moires sont appelées également à présider à la fondation des concours olympiques, comme il convient à des puissances qui peuvent sanctionner à la fois l'accomplissement du rituel inaugural (teletê) et la vie de la nouvelle institution ${ }^{25}$.

Ainsi que le suggère ce rapide aperçu, le profil des Moires est complexe dès l'époque archaïque. Il comprend aussi des compétences d'ordre normatif et politique sur lesquelles nous n'avons pas le temps de nous arrêter ici. Mais revenant au lien des Moires avec le sort du nouveau-né, qui nous intéresse plus particulièrement, il est impératif de mentionner, ne serait-ce qu'en passant, l'histoire bien connue de Méléagre et de sa mère Althaia ${ }^{26}$ : selon certaines versions de cette histoire, les Moires

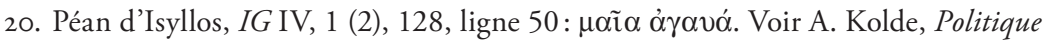
et religion chez Isyllos d'Epidaure.

2I. Pindare, fr. 30 (éd. B. Snell - H. Maehler, trad. d'après A. Puech) : «D’abord la céleste Thémis, la bonne conseillère, sur un char attelé de chevaux harnachés d'or, des sources de l'Océan, fut amenée par les Moires, sur une route brillante, jusqu'aux augustes degrés de l'Olympe pour y être la première épouse de Zeus sauveur; elle enfanta les Heures véridiques, au diadème d'or, aux fruits splendides. »

22. P. ex. Pindare, Isthmiques, 6.16-18.

23. Pindare, Olympiques, 1.25-27.

24. Pindare, Olympiques, 7.62-69. Cf. Eschyle, Euménides, 334-336, où le lachos des Erinyes est aussi sanctionné par la filature des Moires.

25. Pindare, Olympiques, 10.51-52.

26. L'histoire est connue par le poète de l'Iliade (9.526-599), mais les Moires n'y apparaissent pas. La mère de Méléagre maudit son fils en évoquant les dieux souterrains et l'Erinye entend sa prière. Voir dans ce volume l'article de J. Fabre-Serris, «L'histoire de Méléagre vue par Ovide». 
apparaissent en rêve à la mère de Méléagre ou bien lui rendent visite le septième jour après la naissance de l'enfant ${ }^{27}$, sans doute en parallèle avec un rituel lié à l'intégration de l'enfant dans le foyer familial. Les déesses confient alors à Althaia un secret dont va dépendre le sort de Méléagre, qui pourra rester en vie tant que le tison du foyer ne sera pas entièrement consumé. La mère cache jalousement ce tison, mais lorsque Méléagre tue les frères d'Althaia, la femme blessée dans ses liens familiaux brûle le tison fatal dans un accès de colère et met fin ainsi, telle une Moire, à la vie de son propre fils. Derrière le côté tragique de cette histoire, nous entrevoyons une fois de plus les Moires dans leur rôle de divinités présidant à la part de vie qui revient à chacun. La particularité de cette histoire, en ce qui concerne les Moires, tient au fait que les déesses impliquent dans leur action la mère de l'enfant, en lui confiant une tâche sans doute beaucoup trop lourde à porter pour une simple mortelle.

Parfois, les Moires se laissent fléchir ou bien sont trompées dans leurs fonctions. Les récits qui rapportent ces cas exceptionnels s'avèrent tout aussi intéressants afin de mieux cerner le profil des divinités impliquées. Ainsi, Apollon obtient des Moires, une fois qu'il a enivré les déesses, un traitement de faveur pour le héros Admète ${ }^{28}$ : une fois venu le moment de sa mort, il pourra exceptionnellement continuer de vivre si quelqu'un parmi ses proches accepte de son plein gré de mourir à sa place. C'est sa femme Alceste qui se sacrifiera, donnant la part de vie qui lui reste pour compenser la prolongation de celle de son mari ${ }^{29}$. Dans un autre récit qui remonte à la période hellénistique, ce n'est pas un dieu, mais une simple femme mortelle qui se joue des Moires. Le sujet de ce récit, qui nous est transmis par Antoninus Liberalis ${ }^{30}$, est la naissance d'Héraclès, que la déesse Héra veut retarder à tout prix: les Moires et Ilithyie, encore une fois associées, sont assises à proximité de la chambre d'Alcmène et bloquent l'accouchement en tenant leurs mains enlacées. Cette posture est très significative: au lieu de dérouler le fil de la vie, elles le nouent. C'est le mensonge de Galinthias, une amie d'Alcmène, qui dénoue la situation: elle proclame que l'enfant est né malgré tout, et voici que

27. Bacchylide, Epinicia, 5.142-144 (éd. B. Snell - H. Maehler); Diodore de Sicile, 4.34.6-7; ps.-Apollodore, 1.8.3; Pausanias, 10.31.4.

28. Eschyle, Euménides, 727-728.

29. Euripide, Alceste, 150-169; 280-325.

30. Antoninus Liberalis, 29. 
les Moires, se croyant dessaisies de leurs pouvoirs, desserrent les mains dans une réaction de surprise et de mécontentement ${ }^{31}$. Les prérogatives des déesses sont bien entendu intactes, comme le prouve le fait que leur geste provoque la délivrance immédiate d'Alcmène. Ce geste reste efficace, même si Galinthias l'a obtenu par la ruse et l'accord des Moires. Même extorqué, il s'avère être la condition nécessaire et suffisante pour qu'Héraclès vienne enfin au monde.

Ces différents récits placent les Moires en relation directe avec la naissance, ce quatteste leur association avec Ilithyie ${ }^{32}$. Mais certaines traditions montrent bien que leurs prérogatives s'étendent à la prospérité de la famille et de la communauté tout entière. Dans les Euménides d'Eschyle, les Moires sont invoquées à plusieurs reprises par leurs sœurs nocturnes, les Erinyes. Dans cette tragédie, les Erinyes sont en colère contre Apollon, le dieu qui protège le héros Oreste. On se rappellera qu'Oreste a tué sa mère, Clytemnestre, pour venger la mort de son père, Agamemnon. Ce matricide est un crime dont la punition est l'apanage des Erinyes et Apollon bafoue ce droit, comme il avait bafoué celui des Moires à faire mourir Admète, ainsi qu'on l'a vu plus haut ${ }^{33}$. Les Erinyes dirigent ensuite leur colère contre la ville d'Athènes qui, guidée par Athéna, a voté l'absolution d'Oreste. Elles prononcent une malédiction contre la terre attique, dont les terribles déesses se préparent à détruire toute semence, tout espoir de vie, qu'il s'agisse des moissons, des troupeaux ou des familles des Athéniens. Les Erinyes finissent par changer d'avis grâce à l'intervention d'Athéna qui les invite à s'installer dans le pays et leur assure d'importants honneurs cultuels, surtout à l'occasion des mariages et de la naissance d'enfants. Quand, à la fin de la pièce d'Eschyle, les déesses enfin réconciliées bénissent la cité d'Athènes et la terre attique, elles se vouent à en protéger la semence et à assurer la survie de la jeunesse. Dans ce contexte, elles s'associent de nouveau aux Moires, en invoquant de la sorte la protection de leurs sœurs sur la communauté:

3I. Pour un commentaire de cet épisode, voir M. Bettini, Nascere, spéc. p. 56-59 et 92-97.

32. Cette association est adaptée par Platon à l'accouchement de l'âme: Platon, Banquet, 206c-d.

33. Eschyle, Euménides, 723-724. L'Erinye coryphée y invective Apollon: "C'est ainsi que tu en agis déjà dans le palais de Phères: tu persuadas les Moires de rendre immortels des humains.» Cf. ibid., 727-728. 
J'écarte de vous les destins qui vont fauchant les jeunes hommes. Accordez aux vierges aimables de vivre aux côtés d'un époux, vous qui en avez le pouvoir, Moires, filles de notre mère, ô divines gardiennes des lois, qui, fixées dans toute maison, à toute heure y faites sentir le poids de vos présences justicières, vous, de toutes les divinités les plus entourées de respect ${ }^{34}$.

Ainsi, les Euménides associent Moires et Erinyes devenues Euménides, et donc bienveillantes, à la prospérité de la communauté. La part d'honneur qui échoit aux Euménides est directement liée au mariage et à la naissance. Quant aux Moires, elles sont invoquées en tant que divinités des justes normes et sont associées au foyer domestique, à la protection de la jeunesse dans son ensemble et à la garantie d'une continuité de la communauté familiale et politique à travers les mariages. En tant que justes distributrices de la part de chacun, elles agiront en ce sens auprès des jeunes de la communauté athénienne qui pourront ainsi s'unir et se reproduire.

A l'échelle de la cité, cela implique, comme le laisse entendre le chœur, que les jeunes gens ne soient pas "fauchés", c'est-à-dire qu'ils échappent à la guerre, fût-elle extérieure ou civile. C'est précisément ce type d'horreur dont les Erinyes en colère menaçaient les Athéniens avant de s'apaiser sous l'effet de la persuasion d'Athéna. Euripide, à la fin du Ve siècle, associera lui aussi les rituels pratiqués "en l'honneur des Moires et des Déesses anonymes", à savoir les Euménides ou Semnai theai, spécifiant même qu' «il est sacrilège qu'ils soient entre les mains des hommes, mais [qu']ils prospèrent sans exception dans les mains des femmes» ${ }^{35}$. Une telle remarque oriente la réflexion vers des rituels liés à la naissance et à la croissance des enfants qu'il convient maintenant d'aborder.

\section{Honorer pour se survivre: quelques cultes aux Moires}

Les réflexions mises en scène par Eschyle ou par Euripide devaient entrer en résonance avec l'expérience des Athéniens assis au théâtre, mais notre documentation proprement cultuelle est soit lacunaire, soit tardive. Les lacunes sont celles d'une inscription presque contemporaine d'Eschyle,

34. Eschyle, Euménides, 956-967 (trad. P. Mazon, modifiée).

35. Euripide, Mélanippe, fr. 14, 9-29. 
qui évoque un sacrifice aux Moires, à Zeus Conducteur des Moires et à la Terre, probablement lors de la plus importante fête d'Athènes, à savoir les Panathénées ${ }^{36}$. Quant à la source tardive, c'est une notice de lexicographe d'époque romaine attestant que des sacrifices prénuptiaux - sans doute attiques - étaient réservés à Héra Teleia, à Artémis et aux Moires ${ }^{37}$. Le premier des sacrifices entre dans le calendrier officiel de la cité et ouvre sur le large spectre de la prospérité de la communauté, au travers de la progéniture de ses citoyens et des fruits de la Terre ${ }^{38}$. Le second sacrifice fait probablement partie des obligations familiales visant à se concilier la bienveillance d'une série de divinités à la veille d'une nouvelle union ${ }^{39}$. Que ce soit dans un registre public, à l'échelle de la cité, ou privé, à l'échelle de la famille, les Moires sont liées aux transitions essentielles de l'existence humaine. La mise en scène d'Eschyle ne laisse aucun doute à cet égard. Mais les interprétations qu'autorise ce dossier attique sont-elles transposables à d'autres attestations cultuelles en d'autres lieux?

Un culte d'Argolide, dans la région de Sicyone, semble aller dans le même sens, même si notre informateur sur ce point date lui aussi de la période romaine ${ }^{40}$. Pausanias, le voyageur en question, a visité un bois sacré de chênes verts et un temple de divinités que les gens de Sicyone appelaient Euménides, à savoir les déesses qui, chez Eschyle, s'étaient transformées de vengeresses en bienveillantes. Et comme le visiteur est aussi un intellectuel pour qui Athènes est le référent culturel par excellence, il précise que les Sicyoniens appellent Euménides les mêmes déesses que les Athéniens nomment "Vénérables», Semnai theai, ce qui est effectivement leur nom cultuel dans la cité athénienne. Et il poursuit:

36. $I G \mathrm{I}^{3}$ 7, 12 (= LSCG 15).

37. Pollux, 3.38. Voir R. Parker, Polytheism and Society at Athens, p. 440.

38. Une inscription du Pirée, datée du IVe siècle (IG II $4971=L S C G$ 22), réglemente l'offrande de gâteaux propitiatoires aux Moires et stipule qu'elles recevront du miel pour trois oboles et trois oboles. Il est impossible de préciser le contexte de ce qui apparaît comme un petit règlement de culte. Les Moires recevaient aussi le sacrifice d'un porcelet dans la tétrapole de Marathon $\left(I G \mathrm{II}^{2} 1358\right.$, col. B, ligne $28=L S C G 20$; cf. Zeitschrift für Papyrologie und Epigraphik 130 [2000], 45-47, col. 2, ligne 28) et leur desservant(e?) avait son siège inscrit dans le théâtre de Dionysos à la période romaine ( $I G \mathrm{II}^{2} 1092$ B 27 = SEG XII, 95, ligne 46; IG $\mathrm{II}^{2}$ 5137).

39. Les dieux étaient nombreux à s'agiter autour des jeunes fiancés et époux... Voir R. Parker, Polytheism and Society at Athens, p. 439-443.

40. Pausanias, 2.11.4. 
"Chaque année, on leur célèbre une fête d'une journée, en leur sacrifiant des bêtes pleines, et la coutume veut que l'on use d'une libation d'un mélange miellé et de fleurs à la place de couronnes. "Et Pausanias de conclure: "Ils accomplissent la même chose sur l'autel des Moires, qui se situe dans la partie en plein air du bois sacré.»

Ce témoignage est plein d'enseignements. Tout d'abord, les Moires et les Euménides partagent non seulement un même espace sacré, mais aussi le temps de la fête ainsi que son contenu. L'association rituelle, à laquelle les tragédies athéniennes faisaient de simples allusions, se confirme ici sous la forme d'une étroite interaction sacrificielle. De plus, nous nous situons dans le cadre public d'une fête inscrite dans le calendrier liturgique de la cité. C'est donc la communauté au sens large qui est concernée par cette double démarche. Enfin, la nature des offrandes prescrites n'est pas banale. Le sacrifice de bêtes pleines n'est pas une pratique habituelle, si l'on en croit la maigreur du dossier documentaire qui l'atteste ${ }^{41}$, et le destinataire en est toujours une déesse. Sans entrer dans le détail de cette problématique qui a déjà nourri bien des hypothèses, la lecture de l'offrande doit, à notre avis, être relativement littérale: offrir des bêtes pleines à une déesse ou à des déesses est la mise en exergue du bénéfice attendu, à savoir la reproduction. Dans la mesure où le sacrifice grec est un vecteur de communication avec le monde suprahumain, le message est clair. Et comme il s'agit aussi d'honorer la divinité invoquée, consentir à l'abattage de bêtes pleines, c'est offrir un bien vraiment précieux. Les libations miellées, quant à elles, doivent entrer en résonance avec la vertu apaisante de la démarche à l'égard de divinités redoutables étroitement liées au cycle vital ${ }^{42}$. Enfin, les fleurs que les participants substituent à leurs couronnes de feuillage habituelles renvoient à la luxuriance printanière d'une nature "pleine» de promesses. Ce dernier point accentue encore la portée du message véhiculé par l'animal immolé.

En n'oubliant évidemment pas les siècles qui séparent nos attestations, les vers d'Eschyle sur les Euménides et les Moires sont comme la glose poétique des gestes très concrets posés à Sicyone tels que Pausanias les rapporte. Les bienveillantes déesses, dont le pouvoir de destruction reste

4I. S. Georgoudi, «Divinità greche e vittime animali»; J. Bremmer, «The Sacrifice of Pregnant Animals", spéc. p. 156-157.

42. P. Stengel, Opferbräuche der Griechen, p. 178-182. 
toujours virtuellement présent sous l'euphémisme qui les désigne, sont invitées à collaborer avec leurs sœurs, les Moires, pour la continuité de la communauté des Sicyoniens.

Un certain nombre de dédicaces réparties dans l'ensemble du monde grec attestent la vitalité du culte des Moires, mais leur brièveté ne permet guère de circonscrire les attentes des dédicants ${ }^{43}$. Il en est deux, toutefois, qui permettent de voir se nouer le thème de la filature en relation à la naissance des enfants et celui de l'accouchement en tant que terme d'un processus de gestation que les Moires sanctionnent.

La première dédicace est attique et date de la période classique. Elle énumère une série de divinités: Rhapso, la "Fileuse», y reçoit sa place, aux côtés, entre autres, d'Hestia, la déesse du foyer, d'Apollon Pythien, de Léto, étroitement associée à l'accouchement de ses enfants, d'Artémis Lochia et d'Ilithyie, préposées à l'accouchement, et d'une série de divinités liées aux cours d'eau et à leur pouvoir générateur, sans oublier les Nymphes Genethliai, déesses "de la génération " ${ }^{4}$. Dans cette configuration divine centrée sur la naissance des enfants et la continuité du foyer familial, le fil de la vie est sans doute aux mains de Rhapso, dont la fonction est analogue à celle que remplissent les Moires dans le dossier qui vient d'être analysé.

La seconde dédicace nous met en présence d'une famille qui remercie le dieu de Delphes pour la naissance d'un enfant: la volonté du dieu s'est accomplie, dit l'auteur de ce poème sur pierre, grâce aussi à l'action de Lochia Kourotrophos, c'est-à-dire Artémis «accoucheuse» et «nourricière d'enfants", et des Moires teleiai ${ }^{45}$. La présence des Moires en rapport à l'accouchement se précise ici dans l'épiclèse teleia qui leur est attribuée et qui signale leur pouvoir dans l'accomplissement du processus de gestation. Et pour témoigner encore du fait que les œuvres littéraires et les actes rituels n’appartiennent pas à des mondes distincts, mais

43. P. ex. IG IV 2, 540; IGBulg I 2, 305(4); SEG XXIV, 1128; IDélos 2443; IG XII 7, 432 .

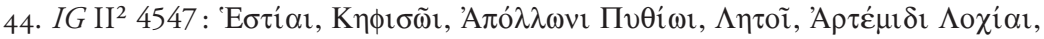

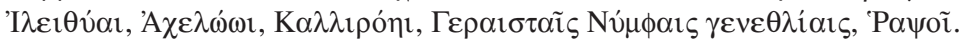

45. Fouilles de Delphes III, 1, 560, v. 7-10 (voir J. Bousquet, "Inscriptions de Delphes», p. 550-557 et fig. 4. Date: 362/1 av. J.-C.; cf. SEG XVI, 341 ; XXV, 586):

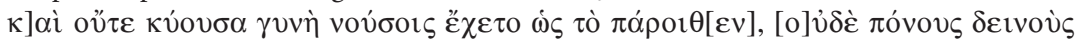

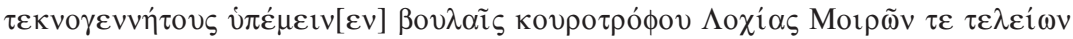
$[\kappa] \alpha[i]$ Фoíßov $\delta i \grave{\alpha} \mu \tilde{\eta} \tau \imath$. 
s'ancrent dans un même savoir partagé, les vers d'Euripide qui chantent la naissance de Dionysos sont comme une exégèse de cette inscription. Le dieu ne vient à la lumière que lorsque sa gestation est parfaitement accomplie, donc à l'issue de cette "seconde" phase qui le voit grandir à l'intérieur de la cuisse de Zeus. Et ce sont précisément les Moires qui signalent que le processus est bien achevé et que le temps de naître est venu ${ }^{46}$. Ces déesses sanctionnent donc à la fois la fin d'un processus, la naissance d'une nouvelle vie et la "part» qui revient au nouveau-né, que ce soit dans un chant en l'honneur de Dionysos, où la "part" est évidemment divine, ou bien dans l'action de grâce d'une famille qui célèbre, au final, la "part» humaine du petit qui lui est né.

Au-delà des dédicaces, souvent brèves, les hasards de la transmission des textes épigraphiques permettent de disposer de deux longues inscriptions de la période hellénistique, respectivement mises au jour à Cos et à Halicarnasse, et qui donnent davantage d'éléments d'interprétation. Le texte de Cos présente les mesures prises par un certain Diomédon, par testament, pour fonder un culte d'Héraclès et des ancêtres, et en assurer la continuité. Différents ajouts sont perceptibles sur la pierre et attestent la vitalité de ce culte familial pendant plusieurs décennies ${ }^{47}$. Sans entrer dans le détail de ce document, le point qui nous concerne se situe au dernier amendement:

Si quelqu'un ose abolir l'une des prescriptions de Diomédôn en causant du tort au culte et aux ancêtres au sujet desquels (135) on a gravé une inscription sur l'autel et sur la stèle, que les enfants de Diomédôn [---] et leurs descendants ne le permettent pas, mais viennent au secours (140) du culte et des ancêtres. Que les epimenioi convoquent l'assemblée, même pour le lendemain, en s’adjoignant qui bon leur semblera. Que les epimenioi soient choisis (145) par les enfants de Diomédôn et leurs descendants. Si un enfant illégitime est reconnu avoir été choisi pour participer au culte, qu'il ne lui soit pas permis de prendre part à la prêtrise. Prenez sur (150) les revenus 50 drachmes pour un sacrifice à Pasios, 40 (pour un sacrifice) aux Moires. Que les descendants du côté masculin sacrifient à Pasios et aux Moires ${ }^{48}$.

46. Euripide, Bacchantes, 99-100.

47. $L S C G 177$ = Iscr.Cos ED 149. I: IVe s. av. J.-C.; II : c. 300 av. J.-C.; III: c. 280 av. J.-C.

48. Traduction Stéphanie Paul. 
Il s'agit donc bien avant tout de continuité familiale. Le fait que des hommes soient engagés dans l'offrande laisse entendre que nous n'avons plus affaire ici au culte féminin des Moires athéniennes évoqué par la Mélanippe d'Euripide et qui était probablement lié à la naissance. Même si l'on se situe dans un cadre privé, ce sacrifice aux Moires est proche, dans sa tonalité et ses objectifs, de ce que les communautés athénienne et sicyonienne prévoyaient dans leur calendrier rituel officiel. La mise en regard du dieu qualifié de Pasios et des Moires est également significative. Pasios est l'équivalent dorien de l'épiclèse ionienne Ktêsios. Or Zeus Ktêsios est le Zeus qui protège le patrimoine de la famille, en permettant la conservation des acquis tout autant que leur accroissement ${ }^{49}$. Lobjectif de cet amendement au testament de Diomédon est clair : il s'agit de se prémunir d'éventuels manquements qui mettraient à mal «le culte et les ancêtres", tout en veillant à la légitimité du détenteur de la prêtrise. Dès lors, on fait appel à Zeus Pasios en tant que protecteur du patrimoine matériel de la famille et aux Moires en tant que protectrices de la continuité de son patrimoine humain. Ce point de vue est encore renforcé par le fait que ce sanctuaire est le lieu où se fêtent les mariages de la famille et que la fondation prend en charge l'aspect financier des mariages de descendants masculins qui n'auraient pas les moyens d'assumer ces frais.

Le document d'Halicarnasse est également un testament, qui sanctionne une fondation cultuelle ${ }^{50}$. Et il est d'autant plus intéressant qu'il s'appuie sur une prescription oraculaire édictée par Apollon:

Quand [Poséidonios] envoya [demander à l'oracle] d'Apollon ce qui serait le plus désirable et le meilleur pour lui-même et ceux advenus de lui, ainsi que de ses descendants masculins et féminins, d'essayer et de faire, le dieu lui répondit qu'il serait plus désirable et meilleur pour eux de propitier et de rendre hommage à Zeus Patrôos, à Apollon qui règne sur Telmessos, aux Moires et à la Mère des dieux, tout comme leurs ancêtres l'ont fait, et également pour eux de propitier et de rendre hommage au Bon Démon de Poséidonios et de Gorgis. S'ils respectent ces ordres, ce sera mieux pour eux.

49. R. Parker, Polytheism and Society at Athens, p. 15-16, 20.

50. LSAM 72, lignes 1-11 = Syll. ${ }^{3} 1044=$ Journal of Hellenic Studies, 16 (1896), p. $234-36, \mathrm{n}^{\mathrm{o}} 36$ (III' $\mathrm{e}$ s. av. J.-C.). 
Le "micro-panthéon" mis en œuvre par l'oracle fait à nouveau intervenir les Moires lorsque surgissent les préoccupations de continuité et de prospérité familiales. Si l'on met à part l'Apollon local auquel la question était adressée, cet ensemble cultuel fait écho, par-delà les siècles, à l'inscription athénienne qui associait Zeus, les Moires et la Terre ${ }^{51}$, de même qu'il évoque la fondation contemporaine de Diomédon à Cos. Zeus et les Moires peuvent ainsi être convoqués pour assurer la continuité d'une lignée familiale, mais aussi d'une communauté au sens large.

\section{Conclusion}

Si nous n'avons guère parlé de "destin " à propos des Moires, ce n'est pas que cette notion eût été hors sujet, mais c'est parce que ce mot, à la fois si chargé et si flou, ne nous dit rien de ce qu'était pour les Grecs ce que nous appelons "Destin». A travers ce parcours aux pays des Moires, en explorant les positions que ces déesses assument autour de la naissance et de la famille, nous espérons avoir contribué à mieux cerner la manière dont les Grecs anciens pensaient le déroulement de la vie humaine, de son début à sa conclusion, et l'enchaînement des événements qui la ponctuent. Les divinités qui y président sont une bonne entrée en matière, pourvu qu'on ne les enferme pas d'emblée dans des étiquettes génériques telles que "divinités du destin». Comme nous l'avons vu, les interventions des Moires, les «Parts» divines, dans la vie des hommes et dans celle de leurs communautés prennent des formes variées, dont la signification ne peut être perçue qu'à l'intérieur de la logique, à la fois concrète et profonde, des cas particuliers, qu'il s'agisse des données des cultes ou bien des représentations narratives. Dans l'un et l'autre corpus, ces figures présentent un point commun, au-delà des éclairages particuliers qui soulignent tel ou tel aspect: elles sont les gardiennes du déroulement de la vie et du cycle vital. Même si d'autres dieux du panthéon grec partagent une telle prérogative, un impact spécifique était pourtant attendu de l'intervention des Moires en cette matière. En vertu de leur lien avec la sphère de la themis et de la dikê, elles déploient les différentes facettes de leur puissance de régulation et de leurs prérogatives en matière de "répartition». Elles peuvent donc être les promotrices bienveillantes du

5I. Voir supra, note 36. 
déroulement de la vie et du cycle vital, mais elles sont aussi les sévères gardiennes des limites qui les conditionnent et les ordonnent. C'est là que se situe leur apanage.

Les Moires règlent la part de vie de chacun, dans la détermination du commencement et de la fin, ainsi que dans les étapes cruciales qui la rythment, dans une juste alternance de biens et de maux. L'image qui s'impose alors est assez facile à saisir sur un plan individuel et bon nombre de dédicaces vont en ce sens. A l'échelle plus large d'une famille, les testaments de Cos et d'Halicarnasse ont montré que les Moires pouvaient intervenir dans le rythme des générations et dans le maintien du groupe à long terme. C'est moins d'une juxtaposition de vies individuelles qu'il est alors question que de la stabilité d'un lignage. Enfin, le culte rendu officiellement aux Moires par les cités, comme Athènes ou Sicyone, atteste qu'une communauté tout entière pouvait se tourner vers de telles déesses, protectrices du cycle vital et d'une juste alternance de biens et de maux. Mais en quoi consistaient précisément de telles attentes à l'échelle d'une cité? Pour répondre à cette question, Eschyle est à nouveau un témoin de choix. Dans le passage des Euménides analysé plus haut, les Moires permettront aux jeunes Athéniens de s'unir et de se reproduire, assurant ainsi le cycle vital de la cité elle-même. Mais on voit aussi se dessiner en ligne de mire le «fauchage des jeunes gens» dont il convient de se prémunir. Dès lors, la juste alternance des biens et des maux au cœur de la polis ne serait-elle pas le juste équilibre entre les naissances et les décès en son sein? La régulation stricte des Moires en cette matière est alors une des conditions de la survie d'une communauté, comme des familles qui la composent.

Vinciane Pirenne-Delforge

F.R.S.-FNRS - Université de Liège

Gabriella Pironti

Université Federico II de Naples 


\section{BIBLIOGRAPHIE}

\section{Abréviations}

IDélos Pierre Roussel, Marcel Launey, Inscriptions de Délos

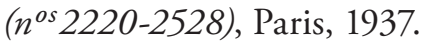

IEG Martin L. West, Iambi et elegi Graeci ante Alexandrum cantati, 2 vols, Oxford, 1989-1992.

IG Inscriptiones Graecae, Berlin, 1903-.

IGBulg Georgius Minailov, Inscriptiones Graecae in Bulgaria repertae, 5 vols, Sofia, $1970^{2}-1997$.

Iscr.Cos Mario Segre †, Iscrizioni di Cos, Roma, 1993.

LSAM Franciszek Sokolowski, Lois sacrées de l'Asie Mineure, Paris, 1955.

LSCG Franciszek Sokolowski, Lois sacrées des cités grecques, Paris, 1969.

SEG Supplementum Epigraphicum Graecum, Leiden, 1923-.

Syll. ${ }^{3} \quad$ Wilhelm Dittenberger, Sylloge Inscriptionum Graecarum, Leipzig, 1915-19243.

Sources

Callimaque, Hymnes - Epigrammes - Fragments choisis, texte établi et traduit par E. Cahen, Paris, Les Belles Lettres, 1922.

Eschyle, Tragédies. Tome II: Agamemnon - Les Choéphores - Les Euménides, texte établi et traduit par P. Mazon, Paris, Les Belles Lettres, 2009.

Euripide, "Mélanippe enchaînée», in Fragments, tome VIII, $2^{\mathrm{e}}$ partie, texte et traduction de F. Jouan et H. Van Looy, Paris, Les Belles Lettres, 2000. 
HésIode, Théogonie - Les Travaux et les Jours - Le Bouclier, texte établi et traduit par P. Mazon, Paris, Les Belles Lettres, 1928.

Homère, Iliade, texte établi et traduit par P. Mazon, 4 tomes, $10^{\mathrm{e}}$ tirage, Paris, Les Belles Lettres, 2002.

Pindare, Isthmiques - Fragments, tome IV, texte établi et traduit par A. Puech, Paris, Les Belles Lettres, 1923.

—, Néméennes, tome III, texte établi et traduit par A. Puech, Paris, Les Belles Lettres, 1967.

—, Olympiques, tome I, texte établi et traduit par A. Puech, Paris, Les Belles Lettres, 1930.

Etudes

Bettini, Maurizio, Nascere. Storie di donne, donnole, madri ed eroi, Torino, G. Einaudi, 1998.

Bianchi, Ugo, $\Delta I O \Sigma$ AIIA. Destino, uomini e divinità nell'epos, nelle teogonie e nel culto dei Greci, Roma, A. Signorelli, 1953.

BousqueT, Jean, "Inscriptions de Delphes», Bulletin de Correspondance Hellénique, 80 (1956), p. 547-597.

Bremmer, Jan, "The Sacrifice of Pregnant Animals», in Greek Sacrificial Ritual. Olympian and Chthonian, ed. by R. Hägg, B. Alroth, Stockholm, Åström, 2005, p. 155-165.

Corsano, Marinella, Themis. La norma e l'oracolo nella Grecia antica, Galatina, Congedo, 1988.

Di Mauro Battilana, Gabriella, "MOIPA» $e$ " $A I \Sigma A$ " in Omero. Una ricerca semantica e socio-culturale, Roma, Ed. dell'Ateneo, 1985.

Dietrich, Bernard C., Death, Fate and the Gods. The Development of a Religious Idea in Greek Popular Belief and in Homer, London, The Athlone Press, 1965.

Georgoudi, Stella, «Divinità greche e vittime animali: Demetra, Kore, Hera e il sacrificio di femmine gravide", in Filosofi e animali nel mondo antico, a cura di S. Castignone, G. Lanata, Pisa, Edizioni ETS, 1994, p. 171-186.

Greene, William Chase, Moira: Fate, Good and Evil in Greek Thought, Cambridge (Mass.), Harvard University Press, 1944.

Kolde, Antje, Politique et religion chez Isyllos d'Epidaure, Bâle, Schwabe, 2003. 
LeClerc, Marie-Christine, "Le partage des lots. Récit et paradigme dans la Théogonie d'Hésiode», Pallas, 48 (1998), p. 89-104.

Mackow iak, Karin, «De moira aux Moirai, de l'épopée à la généalogie: approche historique et poétique de l'autorité de Zeus, maître du destin (Iliade, Odyssée, Théogonie)", Dialogues d'histoire ancienne, 36 (2010), p. 9-49.

Magris, Aldo, L'idea di destino nel mondo antico. I. Dalle origini al $V$ secolo A. C., Udine, Del Bianco, 1984.

Onians, Richard Broxton, Le origini del pensiero europeo, a cura di L. Perilli, Milano, Adelphi, 1998 (éd. or. The Origins of European Thought, Cambridge, Cambridge University Press, 1954).

Parker, Robert, Polytheism and Society at Athens, Oxford, Oxford University Press, 2005.

Patera, Maria, Phobêtra et mormolukeia. Figures de l'épouvante et de la peur dans l'imaginaire grec, thèse de doctorat EPHE, 2004.

—, "Comment effrayer les enfants: le cas de Mormô/Mormolukê et du mormolukeion", Kernos, 18 (2005), p. 371-390.

Pingiatoglou, Semeli, Eileithyia, Würzburg, Königshausen und Neumann, 1981.

Pironti, Gabriella, Entre ciel et guerre. Figures d'Aphrodite en Grèce ancienne, Liège, Centre international d'étude de la religion grecque antique, 2007 (Kernos, supplément 18).

—, «Dans l'entourage de Thémis: les Moires et les “normes” panthéoniques", in La norme en matière religieuse en Grèce ancienne, éd. par Pierre Brulé, Liège, Centre international d'étude de la religion grecque antique, 2009, p. 13-27 (Kernos, supplément 21).

Pistorio, Concetto Bonaventura, Fato e divinità nel mondo greco, Palermo, Tip. M. Greco, 1954.

Rudhardt, Jean, Thémis et les Hôrai. Recherches sur les divinités grecques de la justice et de la paix, Genève, Droz, 1999.

Stengel, Paul, Opferbräuche der Griechen, Leipzig, B. G. Teubner, 1910. 
